Abstract

\title{
First Report on Detection and Genetic Diversity of Picobirnaviruses in the Small Indian Mongoose (Herpestes auropunctatus) ${ }^{\dagger}$
}

\author{
Alyssa Kleymann *, Anne A.M.J. Becker and Souvik Ghosh \\ Department of Biomedical Sciences, Ross University School of Veterinary Medicine, \\ Basseterre, Saint Kitts and Nevis; ABecker@rossvet.edu.kn (A.A.M.J.B.); SGhosh@RossU.edu (S.G.) \\ * Correspondence: alyssakleymann@students.rossu.edu \\ + Presented at Viruses 2020-Novel Concepts in Virology, 5-7 February, 2020; Available online: \\ https://viruses2020.sciforum.net/.
}

Published: 1 June 2020

\begin{abstract}
Picobirnaviruses (PBVs), family Picobirnaviridae, are bi-segmented, double-stranded RNA viruses. PBVs are considered opportunistic enteric pathogens. Gene segment- 1 of PBV encodes the capsid protein, whilst gene segment-2 codes for RNA-dependent RNA polymerase (RdRp). Based on differences in gene segment-2, PBVs are classified into genogroup-I (GI) and GII. Although PBVs have been detected in a wide variety of host species, there are no reports on PBVs from mongoose so far. We report here high rates of detection $(35.36 \%$, 29/82) of GI PBVs in fecal samples from the small Indian mongoose (Herpestes auropunctatus) on the Caribbean island of St. Kitts. Applying a combination of a non-specific primer-based amplification method and conventional RT-PCR using a newly designed primer targeting the 3'-untranslated region (UTR), we could amplify and sequence the complete/nearly complete gene segment-2 of eight mongoose PBV strains. Except for a single strain, the gene segment- 2 of the remaining mongoose PBV strains contained the putative open reading frame encoding the RdRp. The gene segment-2/putative RdRps of the mongoose PBV strains retained various features that are conserved in other PBVs (5'- and $3^{\prime}$-terminal nucleotide sequences, bacterial ribosomal binding site sequence in $5^{\prime}$-UTR, and the three domains in putative RdRps). On the other hand, phylogenetic analysis and sequence identities of the putative RdRps revealed high genetic diversity among the mongoose PBV strains and with those of PBVs from other host species. To our knowledge, this is the first report on detection and genetic diversity of PBVs from the mongoose, expanding the host range of PBVs and providing vital insights into the various features and evolution of putative RdRps of PBVs in a new host species.
\end{abstract}

Keywords: Picobirnavirus; mongoose; gene segment-2; putative RNA-dependent RNA polymerase

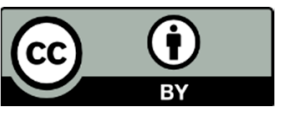

(C) 2020 by the authors. Licensee MDPI, Basel, Switzerland. This article is an open access article distributed under the terms and conditions of the Creative Commons Attribution (CC BY) license (http://creativecommons.org/licenses/by/4.0/). 